\title{
Spatial and spectral regularization for multispectral photoacoustic image clustering
}

\author{
Aneline Dolet ${ }^{1,2}$, François Varray ${ }^{1}$, Simon Mure $^{1}$, Thomas Grenier ${ }^{1}$, Yubin Liu ${ }^{3}$, Zhen Yuan ${ }^{3}$, \\ Piero Tortoli ${ }^{2}$ and Didier Vray ${ }^{1}$ \\ Email: aneline.dolet@creatis.insa-lyon.fr \\ ${ }^{1}$ CREATIS UMR 5220 U1206 \\ Univ Lyon, INSA Lyon, Université Lyon 1 \\ UJM-Saint Etienne, CNRS, Inserm \\ 69621 Villeurbanne, France
}

\begin{abstract}
Photoacoustic imaging is a hybrid modality used to image biological tissues. Multispectral optical excitation permits to obtain functional images thanks to the tissue specific optical absorption that depends on the light wavelength. The aim of this study is to propose a clustering method for photoacoustic multispectral images based on both spatial neighbourhood and spectral behaviour. The proposed methodology is adapted from spatio-temporal mean-shift approach: it clusters distant or neighbouring patterns having similar spectral profiles. The clustering performance of our modified mean-shift algorithm is experimentally tested on multispectral photoacoustic tomography data. Results obtained from phantoms including two blood dilutions and colored absorbers are presented. It is thus shown that our strategy allows the experimental discrimination of media, achieving a clustering performance of more than $99 \%$. Moreover, depending on the applied pre-processing the discrimination of different concentrations of a same medium is possible.

Index Terms-Multispectral photoacoustic, Spatio-temporal mean-shift, Clustering
\end{abstract}

\section{INTRODUCTION}

Photoacoustic imaging is a hybrid method that combines the advantages of acoustic and optical imaging [1]. The modality is based on the detection of acoustic waves produced by a medium submitted to a pulsed laser illumination which increase locally its temperature. Thermal expansions of optical absorbers then create acoustic pressure waves propagating to the media surface where they are detected.

Each tissue has a specific optical absorption that depends on the light wavelength (Fig. 1). Thus, acquiring photoacoustic images at different wavelengths permits to study the spectral profile for each of the media in the region of interest. In particular, in the range 600 to $900 \mathrm{~nm}$, oxygenated (HbO2) and deoxygenated $(\mathrm{Hb})$ bloods have different spectral responses that permit their differentiation [2]. This is, for example, of great interest for the diagnosis of malignant/benign tumours as well as for the follow-up of diseases like carcinoma or for the evaluation of the death of tissues [3].
Different segmentation or classification methods are typically used for multispectral photoacoustic image clustering. Indeed, classic methods such as Principal Component Analysis or Spectral-Fitting were used to identify media in multispectral photoacoustic images [4]. Nevertheless, only few methods have so far considered both spectral and spatial features [5].

The aim of this study is to propose a clustering method of photoacoustic multispectral images that takes into account both the spatial neighbourhood and the spectral behaviour of the pixels. Results obtained from experimental multispectral photoacoustic data acquired on phantoms with two blood dilutions and coloured absorbers are presented.

\section{MATERIALS AND METHODS}

\section{A. Spatial/spectral clustering method}

1) Pre-Processing: The first processing step aims at finding regions of interest in the imaged area. Indeed, a first discrimination between the absorbing media and the background is required. Thus the background is suppressed with a threshold

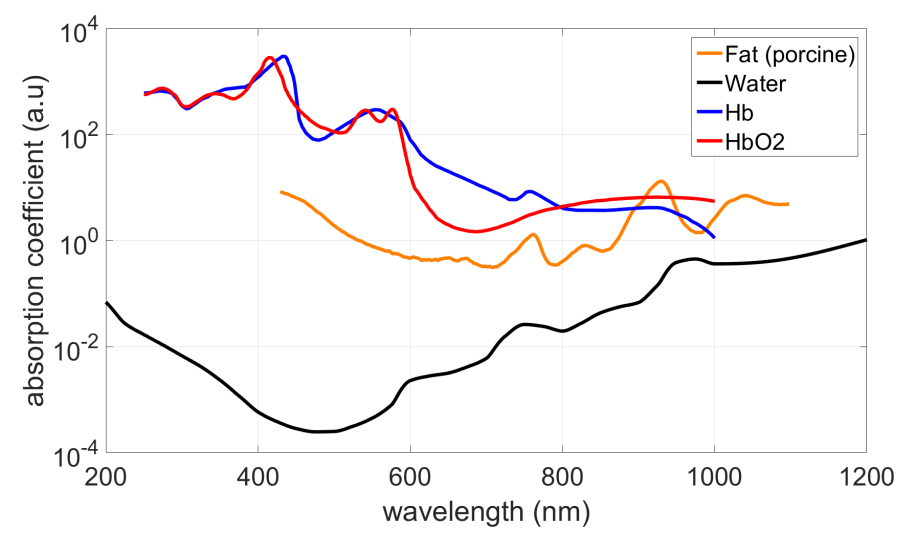

Figure 1. Absorption coefficients of different medias as a function of the wavelength. (a.u.; arbitrary units) 
keeping as region of interest the one with higher acoustic response. The following step depends on the desired strategy, aiming either at discriminating or at merging different concentrations of a same medium. Indeed, changes in the media concentrations imply variations in their spectral profile amplitudes, but not their spectral shapes. Applying a normalization step to the multispectral images will allow to merge the different concentrations of one medium into a same cluster. On the contrary using data without normalization, i.e. the raw data, will allow to discriminate different concentrations as different absorbing medium.

2) Adapted spatio-temporal mean-shift filtering: Meanshift filtering algorithm is based on the maximization of density. An adapted mean-shift algorithm called Spatio-Temporal Mean-Shift (STM-S) was used to cluster longitudinal magnetic resonance images (MRI) [6]. The aim of our study is to adapt this algorithm to multispectral photoacoustic data handling spectral profiles instead of temporal evolution. Multispectral photoacoustic data are obtained from a sequence of 2D images of the region of interest. Each 2D image is acquired at a specific wavelength $\lambda_{l}$ with $l=[1 \cdots L]$ the number of wavelengths. That allows to define a spectral profile $\mathbf{x}_{\lambda, i}$ for each pixel $\mathbf{x}_{i}$ located at a position $\mathbf{x}_{s, i}$. Therefore, the samples $\mathbf{x}_{i}$ that will be used in the iterative filtering procedure are expressed as:

$$
\mathbf{x}_{i}=\left[\mathbf{x}_{s, i} ; \mathbf{x}_{\lambda, i}\right] \in \mathbf{X}
$$

$\mathbf{x}_{s, i} \in \mathbb{R}^{S}:$ spatial position

with $\quad \mathbf{x}_{\lambda, i} \in \mathbb{R}^{L}$ : spectral profile

$i=1, \ldots, N$ : sample index

The idea is, for a given pixel $\mathbf{x}_{i}$, to consider its neighbors $\mathbf{x}_{j}$ in the spatial dimension within a radial distance $R_{s}$ (Fig. 2-left). These pixels $\mathbf{x}_{j}$ satisfy the constraint $g_{s}\left(\mathbf{x}_{s, i} ; \mathbf{x}_{s, j}\right)=1$ where $g_{s}\left(\mathbf{x}_{s, i} ; \mathbf{x}_{s, j}\right)$ is a shorthand notation of $g_{s}\left(\frac{1}{R_{s}^{2}}\left(\mathbf{x}_{s, i}-\mathbf{x}_{s, j}\right)\left(\mathbf{x}_{s, i}-\mathbf{x}_{s, j}\right)^{T}\right)$ with:

$$
g_{s}(a)= \begin{cases}1 & \text { if } a \leq 1 \\ 0 & \text { otherwise }\end{cases}
$$

$R_{\lambda}$ is defined as the maximum distance between the spectral profiles of two samples of one media. Thus pixels belonging to the spatial area defined above that are also spectrally close (controlled by $R_{\lambda}$ ) to the reference spectrum (Fig. 2-right) are used to update, by computing the average, the position and the spectral profile of the reference pixel. Consequently, these pixels also satisfy $g_{\lambda}\left(\mathbf{x}_{\lambda, i} ; \mathbf{x}_{\lambda, j}\right)=1$ where $g_{\lambda}\left(\mathbf{x}_{\lambda, i} ; \mathbf{x}_{\lambda, j}\right)$ is a shorthand notation of $g_{\lambda}\left(\frac{1}{R_{\lambda}}\left\|\left(\mathbf{x}_{\lambda, i}-\mathbf{x}_{\lambda, j}\right)\right\|_{\infty}\right)$ with $g_{\lambda}(\cdot)$ being the same function as $g_{s}(\cdot)$. The spectral iterative meanshift algorithm is then expressed as:

$$
\mathbf{x}_{i}^{[k+1]}=\frac{\sum_{j=1}^{N} g_{s}\left(\mathbf{x}_{s, i}^{[k]} ; \mathbf{x}_{s, j}^{[k]}\right) \cdot g_{\lambda}\left(\mathbf{x}_{\lambda, i}^{[k]} ; \mathbf{x}_{\lambda, j}^{[k]}\right) \cdot \mathbf{x}_{j}^{[k]}}{\sum_{j=1}^{N} g_{s}\left(\mathbf{x}_{s, i}^{[k]} ; \mathbf{x}_{s, j}^{[k]}\right) \cdot g_{\lambda}\left(\mathbf{x}_{\lambda, i}^{[k]} ; \mathbf{x}_{\lambda, j}^{[k]}\right)}
$$
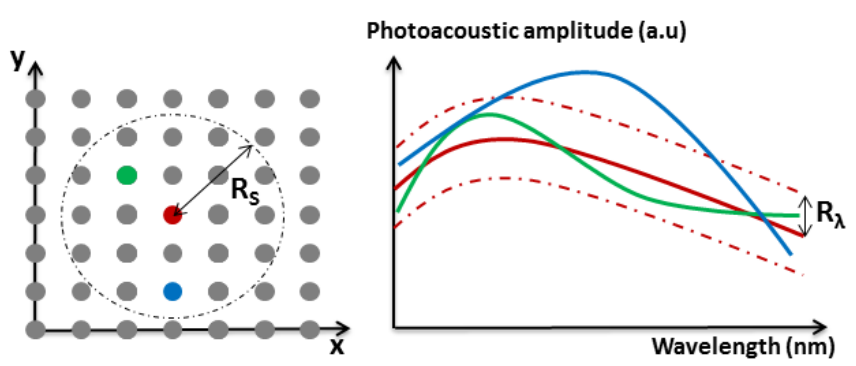

Figure 2. Pixel spatial features at the first iteration with $R_{s}$ parameter (left) and spectral profiles of three pixels with $R_{\lambda}$ parameter (right). The red pixel is the reference one. Even if the green and blue pixels are in the spatial area of the reference pixel, only the green one is used to update the features of the reference. Indeed, the spectral profile of the blue pixel is not close enough to the spectral profile of the reference sample (for at least one wavelength, the blue and the red profiles are separated by a distance superior to $R_{\lambda}$ ). (a.u.; arbitrary units)

These steps are iteratively applied to all pixels $\mathbf{x}_{i}$ of the imaged region $\mathbf{X}$ until no change is obtained in both dimensions between two iterations.

The result of the adapted spatio-temporal mean-shift algorithm can easily be clustered in order to segment the imaged region. All the pixels with close spectral profiles are labelled together (Fig. 3-left). Because of the mean-shift regularization in the spatial domain, two areas of the imaged region can be merged in the same segmentation class even if there are spatially far. Indeed, a same medium might be present at different location in the imaged region.

3) Post-Processing: Due to light diffusion, different areas of the medium having the same absorption coefficient yield photoacoustic signals with proportional values. In the result of the clustering method a media is represented by more than one label because their spectral profiles have strong amplitude differences, even if their shapes are similar (Fig. 3-left). The aim is here to keep only the biggest labelled areas, e.g. those that contain more than $5 \%$ of the photoacoustic image pixels and leaving the others, the bad clustered ones, unlabelled (Fig. 3-right). These labelled areas correspond to the different media to discriminate. Post-processing is done to reduce the number of labels using only the spatial feature in a nearest neighbor
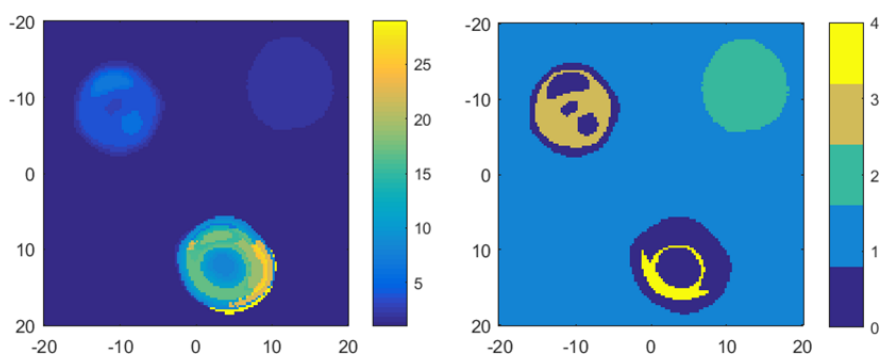

Figure 3. Output of the adapted mean-shift algorithm, e.g. a 2D map showing a segmentation of the imaged region (left). 2D map keeping only the biggest labelled areas, the unlabelled pixels are in dark blue, i.e. label equals to zero (right). 
manner. Considering $\mathbf{Y}=\left\{\mathbf{y}_{i}\right\}_{i=[1 ; C]}$ and $\mathbf{U}=\left\{\mathbf{u}_{j}\right\}_{j=[1 ; N-C]}$ the sets of $C$ labelled pixels and $N-C$ unlabelled pixels, respectively:

$$
\mathbf{y}_{i}=\left[\mathbf{x}_{s, i} ; c_{i}\right] \quad \mathbf{u}_{j}=\left[\mathbf{x}_{s, j} ; c_{j}=0\right]
$$

where $\mathbf{x}_{s, i}$ is the spatial position of the pixel $\mathbf{x}_{i}$ and $c_{i} \in \mathbb{R}$ its associated label. Then for every unlabelled pixel $\mathbf{u}_{j} \in U$, the closest spatial pixel $\mathbf{y}_{i^{*}}$ in $Y$ is found and its corresponding index $c_{i^{*}}$ is affected to $c_{j}$

$$
\begin{aligned}
& \left.i^{*}\right|_{j}=\underset{i=[1 ; C]}{\operatorname{argmin}}\left\|\mathbf{x}_{s, j}-\mathbf{x}_{s, i}\right\| \\
& \text { then } \quad c_{j} \leftarrow c_{i^{*}}
\end{aligned}
$$

\section{B. Experimental data}

The experimental data used in this study have been acquired at the University of Macau using a multispectral photoacoustic tomography (PAT) acquisition scheme presented in Fig. 4(a)

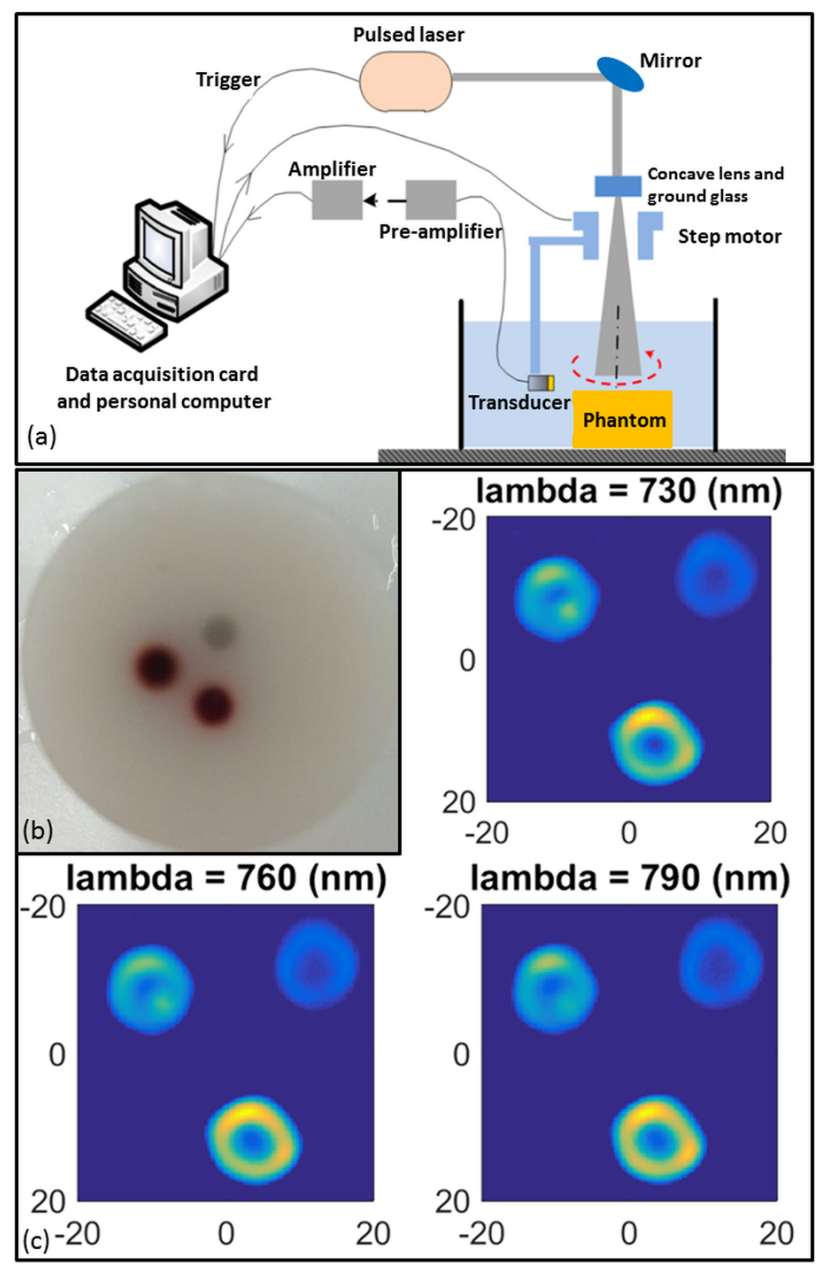

Figure 4. (a) Experimental setup for the photoacoustic tomography acquisitions. (b) Phantom used for the acquisitions, the inclusion in grey is the one of China ink; the two black inclusions contain blood at two different concentrations. (c) Three sample experimental images after thresholding.
[7]. The phantom used is a cylindrical PVA phantom with three spherical inclusions (Fig. 4(b)). There are two inclusions of blood at different concentrations and one of black China ink. The photoacoustic images were acquired at 8 different wavelengths from 700 to $910 \mathrm{~nm}$ (Fig. 4(c)) with $30 \mathrm{~nm}$ steps.

\section{RESULTS}

Two different types of results obtained from the same set of acquired data are presented. The first results are related to clustering without discrimination of concentrations. The second results were obtained by clustering with concentration discrimination, avoiding any normalization step during the preprocessing step.

Multispectral photoacoustic data were processed with the modified mean-shift algorithm presented above. All processing steps are exactly the same except the normalization of the data.

\section{A. No discrimination of cencentrations}

As mentioned before, a normalization step is performed to merge the different concentrations of one medium in the segmentation process. The resulting segmentation map is presented in Fig. 5(c). With such strategy, 100\% of the pixels are well segmented. Indeed, the two inclusions of blood at different concentrations are segmented in the same cluster by the adapted mean-shift algorithm. Note that the background is completely suppressed by the pre-processing thresholding.

\section{B. Concentration discrimination}

To discriminate the different concentrations of blood of our phantom, no data normalization was performed. The corresponding segmentation map is shown on Fig. 5(d). Here, few

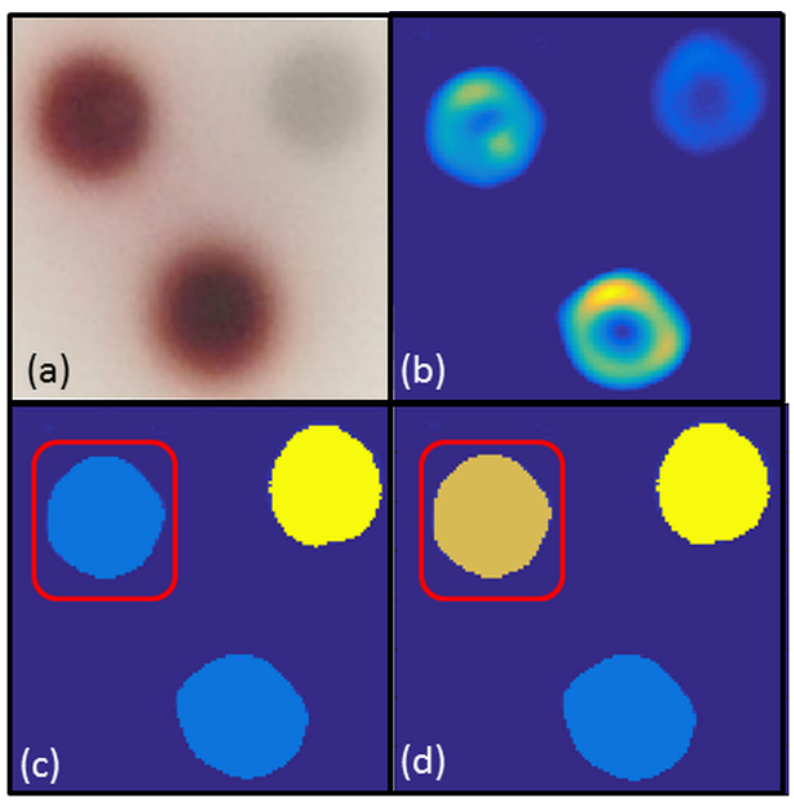

Figure 5. (a) Imaged region of the experimental phantom. (b) PAT of experimental phantom at $730 \mathrm{~nm}$. (c) Result with no discrimination of concentrations. (d) Result with concentrations discrimination. The left up inclusion is clustered with the bottom one if there is no discrimination of concentration or alone if there is a concentration discrimination. 
pixels of the inclusions are segmented with the background by the adapted mean-shift algorithm. It happens because these samples, which are at inclusion borders, exhibit very low photoacoustic amplitude (less than $R_{\lambda}$ ). Nevertheless, the segmentation with concentration discrimination achieves a clustering performance of $99.9 \%$.

\section{CONCLUSION AND DISCUSSION}

A new method for segmentation or classification of media based on multispectral photoacoustic imaging processing has been presented. In particular, it was shown that using the spatial feature to segment multispectral photoacoustic images can increase the clustering performance.

The method used in this study permits to segment multispectral photoacoustic data achieving a clustering performance of more than $99 \%$. Choosing to apply the pre-processing normalization step permits to merge or to discriminate concentration differences of a same medium. Nevertheless, the adapted spatio-temporal mean-shift algorithm needs two parameters to be set up. Choosing these parameters is not an easy task and requires time to find the ones that could give the desired clustering performance. Furthermore, these parameters are probably different for each dataset so that finding the right ones in each case could be time consuming. For the concentration evaluation, by conducting both clustering strategies, the identification of similar media can be obtained and further post-processing could be proposed to estimate the concentration.

The proposed strategy suffers from some limitations, of course. Indeed, with this approach the implicit assumption is that a single optical absorber is present at each pixel. This may not be the case in the discrimination of mixed inks or blood oxygenation quantification, that could be a limitation of our strategy for in vivo applications.

\section{ACKNOWLEDGMENT}

This work was supported by the LABEX CELYA (ANR-10LABX-0060) and LABEX PRIMES (ANR-11-LABX-0063) of Université de Lyon, within the program "Investissements d'Avenir" (ANR-11-IDEX-0007) operated by the French National Research Agency (ANR). It was also supported by the Université Franco-Italienne (VINCI 2016).

\section{REFERENCES}

[1] M. Vallet, F. Varray, M. A. Kalkhoran, D. Vray, and J. Boutet, "Enhancement of photoacoustic imaging quality by using cmut technology: Experimental study," in 2014 IEEE International Ultrasonics Symposium. IEEE, 2014, pp. 1296-1299.

[2] E. R. Hill, W. Xia, D. I. Nikitichev, K. Gurusamy, P. C. Beard, D. J. Hawkes, B. R. Davidson, and A. E. Desjardins, "Interventional multispectral photoacoustic imaging in laparoscopic surgery," in SPIE BiOS. International Society for Optics and Photonics, 2016, pp. 97080B$97080 \mathrm{~B}$.

[3] D. R. Bauer, R. Olafsson, L. G. Montilla, and R. S. Witte, "In vivo multimodality photoacoustic and pulse echo tracking of prostate tumor growth using a window chamber," in BiOS. International Society for Optics and Photonics, 2010, pp. 75 643B-75 643B.

[4] J. Glatz, N. C. Deliolanis, A. Buehler, D. Razansky, and V. Ntziachristos, "Blind source unmixing in multi-spectral optoacoustic tomography," Optics express, vol. 19, no. 4, pp. 3175-3184, 2011.
[5] S. Mallidi, J. Tam, T. Larson, A. Karpiouk, K. Sokolov, and S. Emelianov, "Selective detection of cancer using spectroscopic photoacoustic imaging and bioconjugated gold nanoparticles," in 2008 IEEE Ultrasonics Symposium. IEEE, 2008, pp. 578-581.

[6] S. Mure, T. Grenier, D. S. Meier, C. R. Guttmann, and H. Benoit-Cattin, "Unsupervised spatio-temporal filtering of image sequences. a mean-shift specification," Pattern Recognition Letters, vol. 68, pp. 48-55, 2015.

[7] S. Li, B. Montcel, Z. Yuan, W. Liu, and D. Vray, "Multigrid-based reconstruction algorithm for quantitative photoacoustic tomography," Biomedical optics express, vol. 6, no. 7, pp. 2424-2434, 2015. 